M. Ghaziuddin

I. Al-Khouri

N. Ghaziuddin

\title{
Autistic symptoms following herpes encephalitis
}

Abstract Autism is a childhood onset neurodevelopmental disorder characterized by reciprocal social deficits, communication impairment, and rigid ritualistic interests, with the onset almost always before three years of age. Although the etiology of the disorder is strongly influenced by genes, environmental factors are also important. In this context, several reports have described its association with known medical conditions, including infections affecting the central nervous system. In this report, we describe an 11-year-old Asian youngster who developed the symptoms of autism following an episode of herpes encephalitis. In contrast to previous similar reports, imaging studies suggested a predominant involvement of the frontal lobes. At follow-up after three years, he continued to show the core deficits of autism. This case further supports the role of environmental factors, such as infections, in the etiology of autism, and suggests that in a minority of cases, autistic symptoms can develop in later childhood.

Key words herpes encephalitis autism
1500 East Medical Center Drive

Ann Arbor, MI, 48109-0390, USA

E-Mail: mghaziud@umich.edu

\section{Introduction}

Autism is a neurodevelopmental disorder characterized by a distinct pattern of reciprocal social deficits, communication impairment, and a restricted range of interests. Although most cases are idiopathic in nature, it is associated with known medical conditions in about $10-25 \%$ of cases $[9,18]$. Of these, the role of infectious agents involving the brain is particularly important. Infections that selectively attack certain regions of the brain, not only shed light on the etiology of autism, but also suggest that, at least in some cases, the symptoms of autism may emerge after the first three years of life [8].

Herpes encephalitis is an uncommon but serious infection of the brain. It occurs in 1 in 250,000 to 1 in 500,000 individuals per year but accounts for up to $20 \%$ $\vec{i}$ of viral infections affecting the central nervous system \& [3]. About one third of cases occur in children under 20 years of age [20]. While it usually results in an extremely severe infection, milder forms may also occur. In about a third of cases, the condition is primary, while in the remaining, it results from a reactivation of the virus and is associated with recurrent infections. It is generally accepted that the virus gains entry through the nasal route and lodges in the trigeminal ganglion where it might lie dormant for a long time and, in times of altered immunity, attack the brain. The reason why the virus selectively attacks the temporal and frontal lobe structures has been attributed generally to the physical proximity of these structures to the point of entry of the virus. However, Damasio and Van Hoesen [6] suggested that the encephalitis occurred due to a special affinity of the virus for the limbic cortex. They proposed that the special neuroanatomical and neurochemical properties of the limbic areas involving the temporal and frontal lobes make these vulnerable to the viral infection, regardless of the point of entry, possibly during altered immunological states.

Several behavioral and cognitive symptoms, ranging 
from schizophrenia-like features to movement disorders, have been described following this infection [11]. A few cases of autism have also been reported in both children and adults. DeLong et al. [4] described the occurrence of autism in three children, aged between five and eleven years, who developed autistic symptoms after an acute episode of herpes encephalitis; the symptoms of autism were reported to have resolved completely after recovery. Gillberg [8] described the case of an adolescent with autism. This 14-year-old girl developed autistic symptoms after an episode of herpes encephalitis and continued to show the symptoms long after the acute illness subsided. Ghaziuddin et al. [7] described two males aged between four and eleven years at the time of evaluation whose symptoms started in the neonatal period following an episode of herpes encephalitis. While most studies gave detailed descriptions of autistic symptoms, few have provided information about follow-up and outcome. In this report, we describe the occurrence of autism in an 11-year-old youngster who continued to show the symptoms after three years.

\section{Case history}

PT, an 11-year-old Asian youngster, was referred for a psychiatric evaluation. His father reported that PT was a previously healthy youngster who, about a year prior to the referral, was taken to the emergency room after having developed high fever, sore throat, and increasing drowsiness, while on a camping trip. In addition, he developed a seizure that lasted for about 30 minutes. Serial imaging studies (MRI), performed on admission and repeated after three months, showed bilateral frontal lobe volume loss, especially on the medial sides. The anterior most aspect of the insular cortex was also affected bilaterally. A lumbar puncture was done along with blood and urine cultures. Spinal fluid analysis showed elevated herpes IgG convalescent titers. The IgG titer for herpes simplex type 1 antibody was found to be 13.26 (normal value in our laboratory is 1.00). Based on the clinical presentation, imaging findings and spinal fluid analysis, a diagnosis of herpes encephalitis was made. He was immediately started on a course of intravenous acyclor that lasted for 21 days. His neurological status gradually started improving. However, because of a marked decrease in his self-care skills, he was transferred to the pediatric rehabilitation unit where he stayed for about a month.

About six months after discharge from the hospital, he started showing a host of behavioral problems. These mainly included socially uninhibited behaviors such as hugging strangers, sitting on the lap of the examiner, problems with attention and concentration, periods of hyperactivity and impulsivity, etc. Prior to his illness, PT was described as a good student who was friendly and intelligent with reasonable social skills. A neuropsychological assessment done six months after the initial illness revealed that his level of functioning was now in the moderately retarded range (verbal and performance IQ 56 , full scale IQ 55). In addition, he showed a variable performance on tasks requiring focused attention and freedom from distractibility; and marked problems with executive functioning. Simple tasks requiring psychomotor performance, such as finger tapping, were within normal limits; but more complex tasks which involved a visual component, such as the peg board test, were impaired.

About a year after the initial illness, he was seen for a psychiatric evaluation. The main presenting symptoms were hyperactivity, impulsivity, and a history of increasing 'odd behaviors'. He would walk up to strangers and hug them, and blurt out inappropriate sounds. He would wander around aimlessly, sometimes putting objects in his mouth. His eye contact was poor. The tone of his voice was high pitched. He was described as being lost in a "world of his own". He had difficulty playing in a group with other children his age, and seemed to prefer to be alone. His play was repetitive with little imagination or flexibility. He often spoke only when prompted, and his conversation, when it occurred, was one-sided. He would occasionally repeat phrases out of context, such as "wish you would know, wish you would know". His main preoccupation was flicking channels on the television remote control; when stopped, he would walk over to his neighbor's house to indulge in this activity. In addition, he showed a string of other repetitive behaviors including frequent hand clapping; running his fingers along the edges of the furniture, smelling objects, tapping hard surfaces in a particular manner, etc. Details about his family, birth, and developmental history, were unremarkable. Also, there was no past history of any psychiatric illness. On the Autism Behavior Checklist [15], he scored a total of 89 points meeting the suggested cut-off for autism. On the DSM-IV symptom checklist for autistic disorder [1], he met all the criteria for autism except the onset criterion because he did not have a history of any symptoms before three years of age. However, at the time of evaluation, he showed the typical triad of autism consisting of deficits in socialization, communication and play. The patient was assessed at regular intervals. Despite some improvement in symptoms with the help of behavioral modification and medications, he continued to show the core deficits of autism three years after the index episode.

\section{Discussion}

This patient presented with the classic triad of reciprocal social deficits, communication impairment, and rigid ritualistic interests, all of which emerged after the 


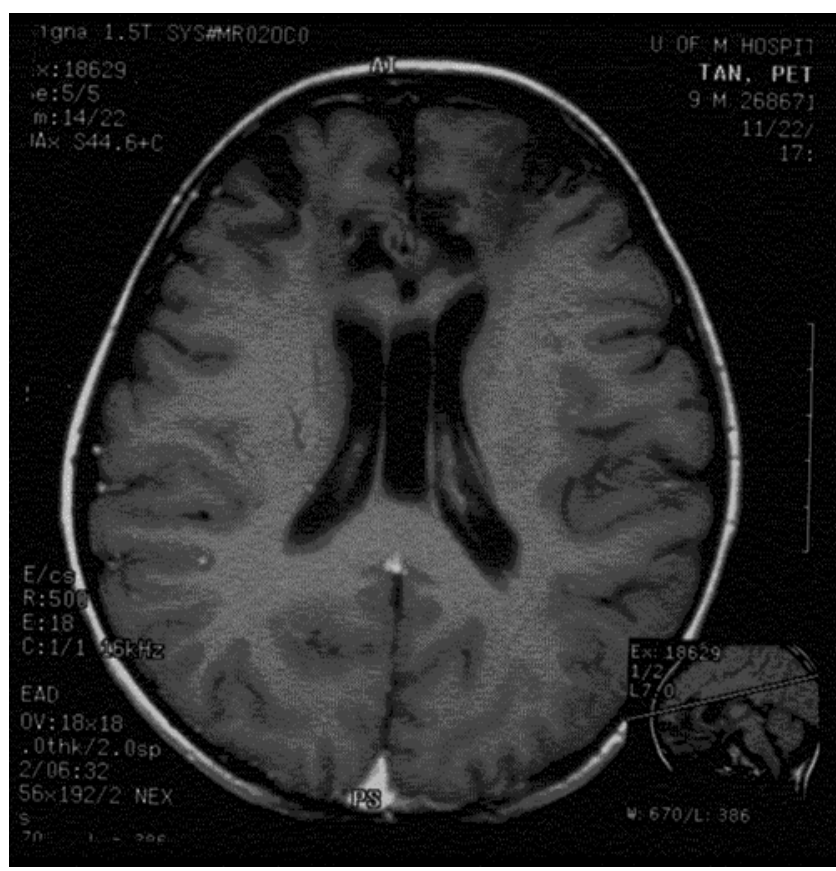

Fig. 1

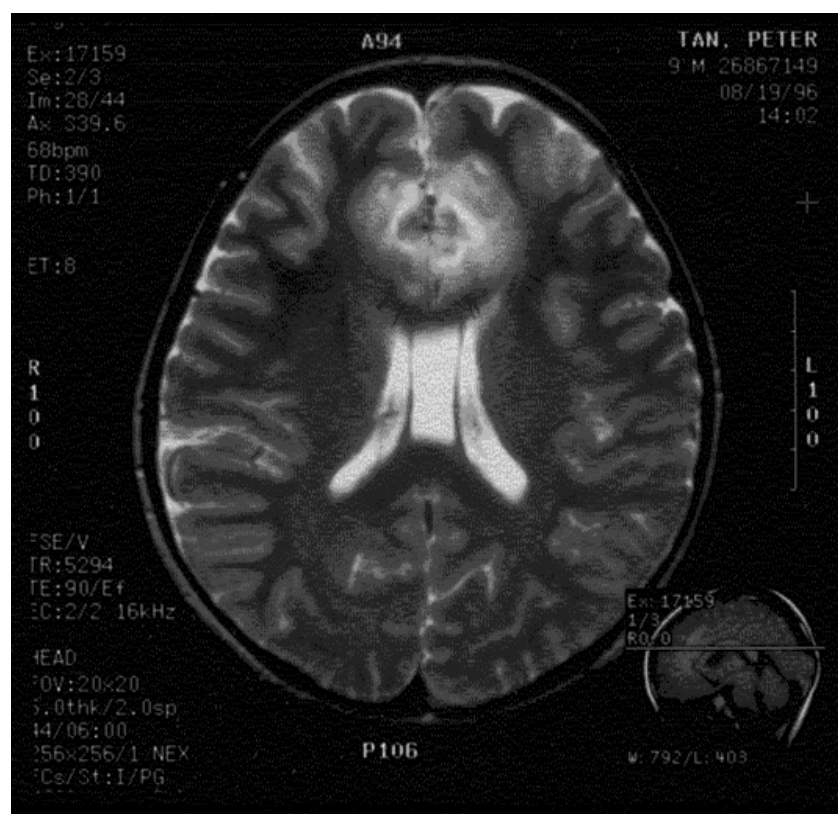

Fig. 2

infection. These symptoms included a failure to develop peer friendships appropriate to his developmental level, and a lack of understanding of the usual social cues. His communication deficits were both verbal and nonverbal, such as a difficulty in initiating and maintaining conversation at the appropriate level, a tendency to ask inappropriate questions of others, problems with eye contact, repetitive use of certain words and phrases, etc. His play was unimaginative and restricted in nature, accompanied by a pattern of repetitive movements such as frequent tapping of the objects in a certain manner. In addition, he showed sensory abnormalities such as a tendency to smell furniture. Since the onset of symptoms was after the age of three years, he was given a diagnosis of pervasive developmental disorder not otherwise specified (PDDNOS) as stipulated in the DSM system of classification [1].

It is important to establish that the symptoms of autism emerged after the infection. The patient had no previous history of any developmental, learning or psychiatric problems. There was no history of head injury. Within months of his infection, he started showing the typical behavioral and social symptoms of autism. Imaging studies also revealed the evolving nature of the infection, and its correlation with the behavioral symptoms. MRI studies performed in the first week of the infection showed evidence of cerebritis or infarction, followed by a gradual spread of the hemorrhagic process to the frontal lobes bilaterally. Three months after the initial infection, the MRI showed bilateral frontal lobe volume loss, especially in the medial frontal structures. In addition, the antibody titers to herpes virus showed a marked increase. Thus, on the basis of the history, the imaging studies, and the laboratory results, it seems very likely that the behavioral symptoms resulted from herpes encephalitis. This report, therefore, is consistent with similar cases described in the literature documenting the close relationship between autism and herpes encephalitis [8].

In addition, the case sheds light on the localization of the virus and the emergence of behavioral symptoms. Typically, herpes simplex virus is said to have a predilection for the temporal lobes. However, other parts of the brain may also be involved and the infection may take the form of meningitis and diffuse encephalitis [13]. Most cases of autism and herpes encephalitis reported in the literature have described damage to the temporal lobes [10]. However, in the present report, damage to the frontal lobes was primary. Longitudinal studies of herpes encephalitis based on MRI studies have found that frontal lobes may also be affected, especially on the medial sides [14]. This suggests that herpes virus does not always affect only the temporal lobes; and that autistic symptoms following herpes encephalitis may involve either the temporal or frontal lobes or both. Indirectly, this report underscores the role of frontal lobes in the etiology of autism and is consistent with similar reports in the literature [5]. For example, individuals with autism display marked deficits on problem-solving tasks, such as the Wisconsin Card Sorting Test, indicating involvement of the frontal lobes [17]. Indirect evidence for the involvement of frontal lobes in autism also comes from the contributions of these lobes to the ability to read 
other people's mental status, so-called theory of mind functions, since this ability is impaired in autism [19]. Delayed maturation of the frontal lobes has been reported in some studies [21], while others have described an increase in the frontal cortex volume that correlates in some cases with cerebellar immaturity in some cases of autism [2].

The patient's symptoms persisted after the initial recovery from the infection. This is consistent with some cases reported in the literature. For example, cases described by both Ghaziuddin et al. [7] and Gillberg [8] showed persisting symptoms of autism long after recovery from the acute infection. However, other reports have described cases in which the symptoms of autism disappeared after recovery from the infection. For example, DeLong, Bean and Brown [4] described three cases, aged between five and eleven years, who developed acute encephalitis, accompanied by symptoms of autism, that resolved after the clinical recovery. It is not clear what type of patients 'recover' from autistic symptoms after the initial infection since the assessment of autistic symptoms in the acute phase of a medical illness is difficult. Also, since autistic symptoms do not always occur in cases of herpes encephalitis, it is important to investigate the reasons why this happens only in a minority of cases.

Autism is generally regarded as a childhood-onset disorder, the cause of which is not known in the majority of cases. Recent research on the genetics of autism has suggested the possibility that the disorder is strongly genetic in nature. About $90 \%$ of the variance in the etiology is said to be caused by genetic factors, the mode of transmission being multifactorial, with several different genes contributing to its etiology. The present case report suggests that despite the genetic loading of this condition, in a small number of cases, the cause could be entirely environmental. In this case, the infection caused by the herpes virus appears to have been the main cause, perhaps the only cause, resulting in the symptoms of autism. The role played by infection in the etiology of autism is unclear. However, several infectious agents have been implicated. Studies from the animal literature have suggested that prenatal exposure to infectious agents and toxins acts by interacting with developing neural and immune elements to create neurodevelopmental deficits. It has been suggested that viral infection with the Borsna virus of Lewis rats may result in deficits of play and social behaviors resembling autism $(12,16)$. In summary, this case describes the emergence of autistic symptoms in a previously healthy youngster following an episode of herpes encephalitis. It provides further evidence that autistic symptoms can sometimes emerge after the age of three years following an external event such as an infection.

Acknowledgements The authors acknowledge the help of the NARSAD foundation.

\section{References}

1. American Psychiatric Association (1994) Diagnostic and Statistical Manual of Mental Disorders, $4^{\text {th }}$ edn. Rev. APA, Washington, DC

2. Carper RA, Courchesne E (2000) Inverse correlation between frontal lobe and cerebellum sizes in children with autism. Brain 123:836-844

3. Corey I, Spear PG (1986) Infections with herpes simplex viruses (second of two parts). New England Journal of Medicine 314:749-757

4. DeLong GR, Bean SC, Brown FR III (1981) Acquired reversible autistic syndrome in acute encephalopathic illness in children. Archives of Neurology 38:194

5. Damasio AR, Maurer RG (1978) A neurological model for childhood autism. Archives of Neurology 35:777-786

6. Damasio AR, Van Hoesen GW (1985) The limbic system and the localisation of herpes simplex encephalitis. Journal of Neurology, Neurosurgery, and Psychiatry 48:297-301

7. Ghaziuddin M, Tsai L, Eilers L, Ghaziuddin N (1992) Brief report: autism and herpes simplex encephalitis. Journal of Autism and Developmental Disorders 22:113
8. Gillberg C (1986) Brief report: onset at age 14 of a typical autistic syndrome. A case report of a girl with herpes simplex encephalitis. Journal of Autism and Developmental Disorders 16:369-375

9. Gillberg C, Coleman M (1996) Autism and medical disorders: a review of the literature. Developmental Medicine and Child Neurology 38:191-202

10. Greer MK, Lyons-Crews M, Mauldin LB, Brown FR III (1989) A case study of the cognitive and behavioral deficits of temporal lobe damage in herpes simplex encephalitis. Journal of Autism and Developmental Disorders 19: 317-326

11. Hargrave DR, Webb DW (1998) Movement disorders in association with herpes simplex virus encephalitis in children: a review. Developmental Medicine and Child Neurology 40: 640-642

12. Hornig M, Weissenbock $\mathrm{H}$, Horscroft $\mathrm{N}$, Lipkin WI (1999) An infection-based model of neurodevelopmental damage. Proceedings of Natl Acad Sci USA 12:12102-12107
13. Kaji M, Kusuhara T, Ayabe M, Hino H, Shoji H, Nagao T (1996) Survey of herpes simplex virus infections of the central nervous system, including acute disseminated encephalomyelitis, in the Kyushu and Okinawa regions of Japan. Mult Scler 2:83-87

14. Kapur N, Barker S, Burrows EH, Ellison D, Brice J, Illis LS, Scholey K, Colbourn C, Wilson B, Loates M (1994) Herpes simplex encephalitis: long term magnetic resonance imaging and neuropsychological profile. J Neurol Neurosurg Psychiatry 57:1334-1342

15. Krug DA, Arick JR, Almond PJ (1980) Behavior checklist for identifying severely handicapped individuals with high levels of autistic behavior. Journal of Child Psychology and Psychiatry 21:221-229

16. Pletnikov MV, Rubin SA, Vasudevan K, Moran TH, Carbone KM (1999) Developmental brain injury associated with abnormal play behavior in neonatally Borna disease virus-infected Lewis rats: a model of autism. Behav Brain Res 100:43-50 
17. Rumsey JM, Hamburger SD (1988) Neuropsychological findings in highfunctioning men with infantile autism, residual state. Journal of Clinical Experimental Neuropsychology 10:201-221

18. Rutter M, Bailey A, Bolton P, Le Couteur A (1994) Autism and known medical conditions: myth and substance. Journal of Child Psychology and Psychiatry 35:311-322
19. Stone VE, Baron-Cohen S, Knight RT (1998) Frontal lobe contributions to theory of mind. Journal of Cognitive Neurosciences 10:640-656

20. Whitley RJ, Soong SJ, Linneman C Jr, Liu C, Pazin G, Alford CA (1982) Herpes simplex encephalitis: clinical assessment. Journal of the American Medical Association 247:317-320
21. Zilbovicius M, Garreau B, Samson Y, Remy P, Barthelemy C, Syrota A, Lelord G (1995) Delayed maturation of the frontal cortex in childhood autism. American Journal of Psychiatry 152: 248-252 